\title{
Assisting Students with Intellectual and Developmental Disabilities in Inclusive Education with Smartwatches
}

\author{
Hui Zheng, Vivian Genaro Motti \\ Department of Information Sciences and Technology \\ George Mason University \\ Fairfax, USA \\ \{hzheng5lvmotti\}@gmu.edu
}

\begin{abstract}
Smartwatches have a large potential to support everyday activities. However, their potential as assistive technologies in inclusive academic environments is unclear. To investigate how smartwatches can support students with intellectual and developmental disabilities (IDDs) to perform activities that require emotional and behavioral skills and involve communication, collaboration and planning, we implemented WELI. WELI (Wearable Life) is a wearable application designed to assist young adults with IDDs attending a postsecondary education program. This paper reports on the user-centric design process adopted in the development of WELI, and describes how smartwatches can assist students with IDDs in special education. The results reported are drawn from 8 user studies with 58 participants in total. WELI features include behavioral intervention, mood regulation, reminders, checklists, surveys and rewards. Results indicate that several considerations must be taken into account when designing for students with IDD, and that overall the students are enthusiastic about adopting an innovative smartwatch application in class, as they reacted positively about the technology and features provided.
\end{abstract}

\section{ACM Classification Keywords}

K.4.2 Computers and Society: Social Issues-Assistive technologies for persons with disabilities; H.5.2 Information Interfaces and Presentation (e.g. HCI): User Interfaces-Usercentered design; K.3.2 Computers and Education: Computer and Information Science Education-Self-assessment

\section{Author Keywords}

Wearables; Intellectual and Developmental Disabilities (IDDs); Assistive Technologies; Smartwatches; Mobile.

\section{INTRODUCTION}

Intellectual and developmental disabilities (IDDs) are characterized by individual limitations in reasoning, learning, prob-

\footnotetext{
Permission to make digital or hard copies of all or part of this work for personal or classroom use is granted without fee provided that copies are not made or distributed for profit or commercial advantage and that copies bear this notice and the full citation on the first page. Copyrights for components of this work owned by others than the author(s) must be honored. Abstracting with credit is permitted. To copy otherwise, or republish, to post on servers or to redistribute to lists, requires prior specific permission and/or a fee. Request permissions from permissions@acm.org.

CHI 2018, April 21-26, 2018, Montreal, QC, Canada

(c) 2018 Copyright held by the owner/author(s). Publication rights licensed to ACM ISBN 978-1-4503-5620-6/18/04 . .\$15.00
}

DOI: https : //doi .org/10 . 1145/3173574 . 3173924 lem solving and behavioral regulation. IDDs are chronic and lifelong. They not only affect a range of everyday social and practical skills of individuals [23], but also severely impact their cognitive and physical abilities. In the U.S., IDDs affect 1 in 6 children [2], [16]. Even when attending inclusive programs, students with IDDs face several challenges in their education. Difficulties arise in processing complex information in the classroom, comprehending course content and completing coursework. Inherent limitations in cognitive and adaptive functioning as well as the formal structure that characterizes educational programs are the main causes for such difficulties. Despite these challenges, pursuing post-secondary education is important for students with IDDs, enabling them to learn, become more independent, acquire employment skills, and experience university life.

To facilitate the integration of students with IDDs, universities must provide these young adults a supportive academic environment $[21,20,11,28]$. To that end, university programs traditionally rely on personal assistants, individuals who provide personalized support for students with IDDs by helping with class contents, coursework, learning activities, as well as monitoring student progress, intervening and mediating when necessary $[12,19]$.

Most special education programs rely on one assistant to help each student with IDD. To offer in-class assistance, the assistant has to be closely located to the student, continuously observing him/her and intervening when necessary. The interventions aim at keeping the students focused, encouraging their participation in class, and mediating their communication and regulating their behavior. The support of the assistant is essential to improve the academic performance of students with IDDs. Nevertheless, as the students with IDDs attend regular classes, the interventions given by assistants are verbal and loud, which can distract the classmates, disturb the instructors and even make the students with IDDs feel uncomfortable due to the potential stigmas.

To investigate how smartwatches can assist students with IDDs in class, we implemented WELI. WELI requirements were elicited through a user-centered design approach, including 8 user studies conducted with 58 participants, among which 37 were assistants and 21 were students with IDDs. The user studies were conducted with the collaboration of three coor- 
dinators of Mason LIFE, a post-secondary special education program. The studies spanned a 2-year period and focused on understanding specific smartwatch features that have potential to assist students with IDDs in their education and classroom experience. As a result, WELI has been designed, developed and evaluated. WELI (pronounced as "Well I") is a mobile and wearable $(\mathrm{M} \& \mathrm{~W})$ application that by assisting students with IDDs aims at facilitating the work of their assistants. By using WELI, the students receive timely interventions and personalized notifications on a smartwatch.

WELI features include: prompt interventions to remind students to focus, to mediate their participation in class, to provide rewards and countdown before a class or a break ends, to send notifications about events and procedural checklists, and to deliver an after-class survey. The application encourages students to be more engaged and collaborative in class, besides facilitating their preparation before classes start. The application also supports students to be focused on their tasks, reinforce their positive behaviors, facilitate self-assessment of affective states and behavioral regulation. For the assistants, these features can enhance their effectiveness in monitoring students and tracking their progress with a more automated and unobtrusive approach which is able to record and monitor self-assessment responses and students feedback. By sending automated notifications, the application can reduce the assistants' workload and provide more consistent assistance to students.

\section{RELATED WORK}

Different solutions have been designed to assist people with intellectual and developmental disabilities, ranging from wearable assistive technologies to mobile applications with games and augmented reality.

\section{Assistive Technologies for Individuals with IDDs}

MOBERO is an assistive mobile application that provides activities and support for parents of children with Attention Deficit Hyperactivity Disorder (ADHD). The application rewards the children, and assists families in establishing morning and bedtime routines that are healthier and more effective for the children [27]. A web-based learning system for iPad was implemented to help Individuals with Developmental Disabilities (DD) to learn basic mathematical skills [18, 3]. It supports individuals with DD of all ages by providing activities that train basic skills, including seven exercises with numbers and uppercase and lowercase letters, colors, shapes and U.S. currency.

Incloodle is a picture-taking iPad application in which two children play together using a single device. This game aims at promoting inclusion for children with and without disabilities [26]. Four versions of the application are available, varying in cooperation levels and characters. The game aims at lowering barriers by encouraging inclusion. GazeSpeak is a smartphone application with an eye-tracking communication system developed to help individuals with motor disabilities, especially patients with Amyotrophic Lateral Sclerosis (ALS). GazeSpeak recognizes and interprets eye gestures in real time, decoding these gestures into predicted utterances, to facilitate communication. Different user interfaces are available for speakers and interpreters [31].

Prior research on assistive games include a Kinect-based solution developed to support physical rehabilitation for young adults with Cerebral Palsy (CP) [5]. This game provides training for movements necessary to self-feeding and self-dressing. A Kinect-based system is employed to detect the users' poses in the game. To promote upper limb rehabilitation, the game determines whether the movements executed correspond to the therapists' expectations and provide feedback accordingly. Kirana is a Kinect-based application designed to simulate real world shopping scenarios aimed at improving life skills and promoting self-efficacy for individuals with developmental disabilities (DD). By buying groceries from a local store, this solution integrates arithmetic and social interaction, breaking down complex tasks into several social, mathematical and decision-making activities performed through gesture-based interactive game actions [24].

Exergames were designed to support children with motor coordination disabilities occasioned by developmental disorders. These exergames enable users to practice gross and fine motor coordination skills, focusing on self-care activities [4]. A virtual ethnographic study of Autocraft was performed to understand how do-it-yourself and appropriation in games can have assistive properties and therapeutic uses for the youth with autism. Using a whitelisted Minecraft game server for young adults with autism and their colleagues [22], this work examines how this community benefits from the platform, where the players use 'mods', a do-it-yourself software in Minecraft, to transform features of the virtual world and provide a variety of assistive technologies. It supports the users with self-regulation and socialization activities by also involving other users.

Following the trend of augmented reality, assistive technologies have been explored to support cognitive impairments. To enable individuals with cognitive impairments to work with efficiency in sheltered organizations (facilities that employ primarily individuals with disabilities), an assistive system for the workplace has been created [10]. It uses a top-mounted projector and a top-mounted Kinect depth camera, providing in-situ projected context-sensitive instructions to the worker. Through continuous feedback shown by green lights, individuals with cognitive impairments receive information about the activity steps related to the product assembly.

\section{Assistive Wearables}

As wearable devices have become more prevalent in the last few years, they have begun to be explored as assistive technologies. The variety of existing form factors, sensors available for data collection and large potential for prompt and customized notifications make wearable solutions well-suited to provide support for multiple disabilities. To assist users with visual impairments, a Google Glass application has been implemented. By providing navigation aids, this application [8] helps blind users to find doors in large open spaces. Freeman et al. (2017) studied novel ways of using sound from wrist wearables for audio output [9]. Using proximity estimation, this technology helps children with visual impairments in school. To 


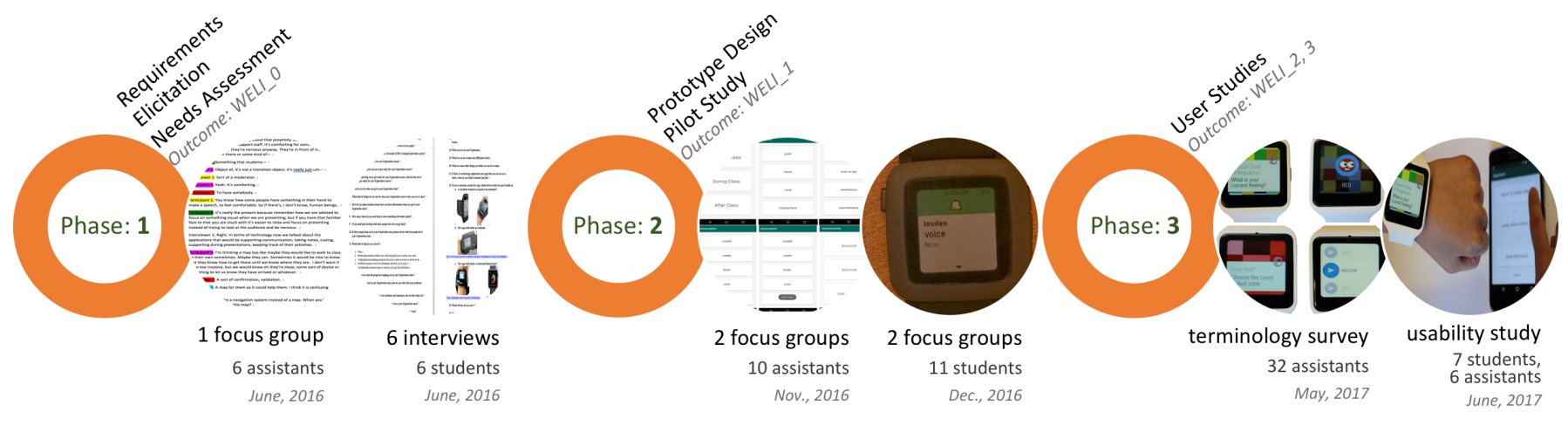

Figure 1. The implementation of WELI followed an iterative user-centered design approach structured in three phases. The project combined focus groups, interviews, and usability studies with assistants and students with Intellectual and Developmental Disabilities (IDDs).

help users with gait impairment, GaitAssist employs several on-body wearable inertial motion units and a smartphone to provide real-time feedback to patients whenever freezing of gait occurs [17].

Few wearable technologies have been explored to assist users with cognitive disabilities and developmental disorders. ProCom facilitates proximity awareness in social communication for individuals with autism using chest-worn wearables [1]. ProCom uses an infra-red sensor module worn on the user's chest and connected to a mobile application to show the changes of distance and zones of proximity. Making use of augmented gloves, a study conducted by Kosch et al. (2016) compared the tactile, auditory, and visual modalities to provide error feedback to workers with cognitive impairments [14]. The augmented gloves are equipped with vibration motors to provide tactile feedback when an error occurs.

Among the existing systems that explore the use of wrist-worn technologies to assist users with cognitive and developmental disabilities, we highlight EnhancedTouch, Snap, FOQUS and WatchMe. EnhancedTouch is a bracelet with LED lights that measures human-human touch events and provides visual feedback to augment human-human interaction via physical touch. EnhancedTouch focuses on supporting communication for children with autism [29]. Snap is a personalized wristband developed through participatory design. Snap helps to analyze anxiety levels of adults with Autism by recording the user interaction with a digital stretchy wristband [25].

FOQUS is a smartwatch application that aims at reducing anxiety and stress of adults with ADHD and mild forms of attention deficit disorders by promoting focus. The features available include Pomodoro for time management, guided meditation, and positive message priming [6]. WatchMe is a wearable application designed to detect behavioral problems and manage those through interventions, helping in behavioral regulation for children with neuro-developmental disorders [30]. Interviews with adults with mild cognitive impairments working in a sheltered workshop (a facility that employs primarily individuals with disabilities) were conducted to explore the potential of wearables to address special needs related to health, family, and productivity [7].

\section{USER-CENTERED DESIGN}

Following an iterative user-centered design approach structured in three phases (Figure 1), we designed and developed WELI. WELI is a wrist-worn solution for students with IDDs following inclusive post-secondary programs. After the IRBapproval of the study protocol, we conducted eight user studies. The study participants included 37 assistants and 21 students with IDDs.

\section{Phase 1: Requirements Elicitation}

To understand the potential of smartwatch applications to support students with IDDs, three main factors must be considered: (1) the activities executed by assistants when providing support to the students, (2) the scenarios and contexts in which each activity takes place and (3) the potential of wearable technologies in facilitating and enhancing such activities.

To gain knowledge about these factors, we conducted a 90minute focus group with six assistants experienced in Special Education. The six assistants worked for Mason LIFE, a U.S.-based inclusive post-secondary education program for students with IDDs. The study included one professor, who is the program director and has 25 years of experience in special education; one program coordinator, who is also a licensed special education teacher with ten years of experience in special education; and four assistants who regularly assist young adults with IDD in inclusive classes. Each assistant had about one year of experience working in the program.

In this focus group, the topics covered the daily activities of the assistants, including the type of support provided to the students in class, the approach employed to provide support, the context where the students were assisted, the aspects in which the support helped the students, the difficulties faced in executing these activities, as well as preferred approaches for assisting students. The focus group also discussed the application of wearable technologies to the support of students with IDDs, including how students would react to, accept and adopt wearable technologies; the activities that wearables could possibly improve or augment to help these students; the scenarios and context in which wearables could be used; inherent challenges; and the preferences concerning the specification of a wrist-worn device (e.g. color, size, design, interaction and functionality). 
After the focus group, to understand and confirm the requirements with the viewpoint of students, we conducted six semistructured interviews (each lasting about 60 minutes) with six students with IDDs from the Mason LIFE program. As trust is very import in work with students with IDDs [13], these interviews were conducted by the coordinator of the program, with whom the students are already familiar. The interview script covered questions about what and how the students perform in class, when and how often they need help from the assistants, what type of help they need, how they receive help, what technology they use and how they use the technology, which kinds of wearable devices they prefer, and how far from the students the assistants are located in class.

\section{Findings}

Combining the analysis of results from the focus groups and interviews, we identified design considerations, directives and key requirements to implement a wearable application for special education.

From the focus groups, it became clear that the goal of WELI was not to replace the assistants with a technological solution, but to facilitate the support they provide to students in class to becomes less obtrusive. Not only is the help from the assistant essential to students but also some of their activities (related to observing the students' behavior and taking notes) can not be fully automated due to technological limitations. Therefore, the ultimate goal of WELI is to facilitate the inclusion of students with IDDs in class, aligned with the mission of inclusive post-secondary education to enable students to cultivate a sense of independence and offer them a valuable university experience, so that they can practice the necessary skills for a smoother transition to the job market. As one of the coordinators pointed out in the focus group:

“... there are evidences to say that having an assistant can cause a barrier to our students to be fully integrated in class. So that it is one of the things that wearable technology can try to compensate for. How can we provide the support but not have the person barrier? There are a lot of things that the person [assistant] does which is good. But at the same time, just having that person signals that the students are different. It signals that they need additional help. It signals that many times another student would talk with the assistant before they are going to talk with the student. Those are some of the issues in sending a person, even a person who is doing absolutely everything correctly."

Moreover, both the assistants and the students mentioned they preferred the assistants to sit farther away at the back of the classroom and not beside the students, as this would allow them to become more independent and also more integrated with their peers in class.

For the acceptance of wearable technologies, we found that there were no important concerns for the students to accept and learn how to use smartwatches, as all students were excited about novel technologies besides also being familiar with technology in general and experienced with smartphones. Although all the six students interviewed reported to know about smartwatches, only one among them already had a smartwatch. He also knew how to use it and used it regularly. Concerning the non-functional requirements for wearable devices, the students and the assistants emphasized the need for a device that was sturdy as well as waterproof.

The main functional requirements that WELI should support in class are aligned with the current duties of the assistants. Five primary features we identified are: (1) maintaining the student focus and calm, (2) helping students to communicate, collaborate and participate in class, (3) moderating their voice volume when they speak, (4) intervening to regulate behaviors that are less appropriate in class, and (5) helping them with the timing of when to question, listen or stop talking. For supporting students outside the classroom, we identified three main needs to support: checking the class materials before classes start, checking the class contents to prepare for new vocabularies, and reminding the students about assignments and homework.

\section{Phase 2: Design of the Prototype and Pilot Study}

Given the main goals, requirements, and users preferences identified in the studies as presented in the previous section, we built a WELI prototype for preliminary assessment in a pilot study. WELI 0 (Wearable Life version 0 ) was designed and developed as a mobile and wearable application for Android phones, using Nexus $5 \mathrm{x}$ and the Pebble Time smartwatch. The devices were connected via Bluetooth.

As the key requirements drawn from phase 1 aimed at reducing the obtrusiveness of "a personal assistant" in class, we focused on implementing and assessing the set of features for in class scenarios. WELI 0 was equipped with basic intervention features, as listed in phase 1 and described in detail in [32] and [33]. The application goals were notifying the student to keep himslef/herself focused, to ask or answer questions, to participate in group discussions and to moderate his/her voice (speaking more or less loudly). By using the mobile phone version of the WELI application, the assistant selects one feature among the options available on the main menu. The notifications are delivered directly on the student's watch, starting with a short vibration to grab the student's attention. The vibration is followed by the display of a graphic and textual information corresponding to the feature selected by the assistant. The maximal range supported by Bluetooth connections is 100 meters, which allows the assistant to sit in the back of classrooms with their phones to remotely monitor students and send interventions.

To gather feedback and suggestions on our initial prototype, we conducted four focus groups of 60 minutes each, involving the assistant as well as students. In each session, the initial version of WELI was demonstrated on the actual devices and through a video. The video highlighted the features implemented in the prototype in more detail, presenting the user interface and demonstrating the communication between the phone and the smartwatch. The studies aimed to gather the users' opinions about the design of the prototype, refine the users' requirements, identify new features to be added to the application, and understand the context of use for the features presented. Among the four focus groups, two were conducted 


\begin{tabular}{|c|c|c|}
\hline Theme & Keywords & Frequency \\
\hline Device & $\begin{array}{l}\text { watch, smartphone, durable, } \\
\text { water-resistant, trendy, fashion- } \\
\text { able }\end{array}$ & $22(13)$ \\
\hline Graphics & $\begin{array}{l}\text { like images, add pictures, vi- } \\
\text { sual support }\end{array}$ & $21(7)$ \\
\hline Preparation & prior class, materials, checklist & $18(8)$ \\
\hline Wearable & $\begin{array}{l}\text { wear, usage, maintenance, du- } \\
\text { ration, storage, accessibility }\end{array}$ & $18(11)$ \\
\hline Participation & $\begin{array}{l}\text { encourage, talk, discuss, ask, } \\
\text { answer, question, timing, fre- } \\
\text { quency, content }\end{array}$ & $15(9)$ \\
\hline Quiet & $\begin{array}{l}\text { when, stop talking, unrelated, } \\
\text { listen }\end{array}$ & $15(8)$ \\
\hline Focus & listen more, message & $14(10)$ \\
\hline Classroom & $\begin{array}{l}\text { inclusive, independence, barri- } \\
\text { ers, non-disabled student }\end{array}$ & $9(5)$ \\
\hline Regulation & zones, mood & $7(0)$ \\
\hline Break & drink water, take, need & $7(1)$ \\
\hline Reminder & schedule, calendar & $7(2)$ \\
\hline Customize & specific, universal, personalize & $2(0)$ \\
\hline Homework & Blackboard & $1(1)$ \\
\hline
\end{tabular}

Table 1. Main themes discussed in the focus groups, set of key words associated with each theme, and the frequency of occurrence

with five assistants each; and the other two were conducted with the students with IDDs, totalizing 11 participants (four in the former and seven in the later session) of a gender-balanced sample of six female and five male participants.

\section{Findings}

To identify the features necessary in the application and their relevance, we analyzed the contents of the video and the audio transcripts from the focus group, and quantified the frequency of occurrence of the topics discussed. Table 1 summarizes the results of the analysis for all five focus groups.

The frequency of occurrence for a given topic, drawn from the initial focus group conducted in Phase 1, is indicated between parentheses. The results provide a blueprint for the application design, guiding the technical development to meet functional and non-functional requirements according to specific users' needs. The features initially implemented were extended with additional requirements identified in the follow up studies. Additional features included: "preparation before class" (mentioned 18 times), "zones of regulation" (mentioned 7 times), "a time break" (mentioned 7 times), "reminders" (mentioned 7 times) and "customization" (mentioned 2 times). In the focus groups, the participants also confirmed the relevance of all the features previously implemented in WELI.

Concerning design preferences for the graphic user interfaces (GUIs) of the watch, the main recommendation was to ensure that all notifications displayed on the watch screen for the students presented a meaningful background image along with a text message to provide visual cues (mentioned 21 times). Concerning the GUIs for the mobile application for the assistants, we presented and discussed two possible menu layouts-one with a list of buttons and another with tab items. The assistants preferred the UI with a button list.

Concerning non-functional requirements for the devices, analyzing contents related to the key words "device" and "wearable", we identified the need for a durable, water-resistant, trendy and fashionable smartwatch, easy to use and to maintain as well. The storage capability should be enough to record the user interactions during one week, and the battery should also last one week. The assistants would be responsible for recharging the batteries of the smartwatches weekly, as the students were likely to forget to check the battery status often. The device screen should support colored, high-resolution graphic images (mentioned 21 times). Three additional features identified in this phase included completing a "checklist" for "the student preparation before classes start", self-assessing of "mood" for the "zones of regulation", and notifying the student about "a time break".

\section{WELI Re-design}

The Pebble Time was discontinued in the end of 2016. To implement the feedback from the pilot study and re-design WELI, we selected a new smartwatch. Guided by a needs assessment conducted for WELI, we selected the Sony Smartwatch SW50. To implement customized applications, this device is compatible with the Android Wear development framework. Also, both the hardware specification of this watch as well as its flexibility for application development were considered appropriate to address WELI requirements. Considering requirements related to graphic design, the Sony Smartwatch has a transflective screen with a larger dimension (1.6 inch) and better pixel resolution ( $320 \times 320$ pixels) when compared with the Pebble Time (electronic paper screen of 1.25 inch), making it well-suited for the presentation of the graphic icons and background images of the user interfaces.

For interaction needs, including mood regulation and checklists, the Sony Smartwatch provides touch screen capabilities facilitating micro interactions (e.g., swiping, scrolling and tapping) for the students to quickly input their feedback. In contrast, Pebble Time had only three small physical buttons to enable input entry. The Sony watch is also water-resistant and more fashionable due to interchangeable wristbands (multiple color options are available). It has a 2-day battery life for continuous use (considered a relatively long duration among most watches compatible with Android development) and it has 4 GB of internal memory. The Android Wear technology is updated regularly and easier to employ due to its large popularity, frequent updates and detailed documentation.

WELI 1 was implemented as an extended version of the original application to accommodate the additional features. Designed for the Sony Smartwatch, WELI 1 included the original features (i.e. focus, discussion and voice moderation) as well as adding a 'checklist' feature to help students be prepared before class; a 'mood' feature to ask the student to self-assess his/her affective state (self-regulation); a countdown feature to 'remind' a student to come back before a class 'break' ends; an alarm feature to 'remind' the students about an event (for example notifying him/her five minutes before a class ends); and a 'setting' feature to enable users to customize the notifi- 


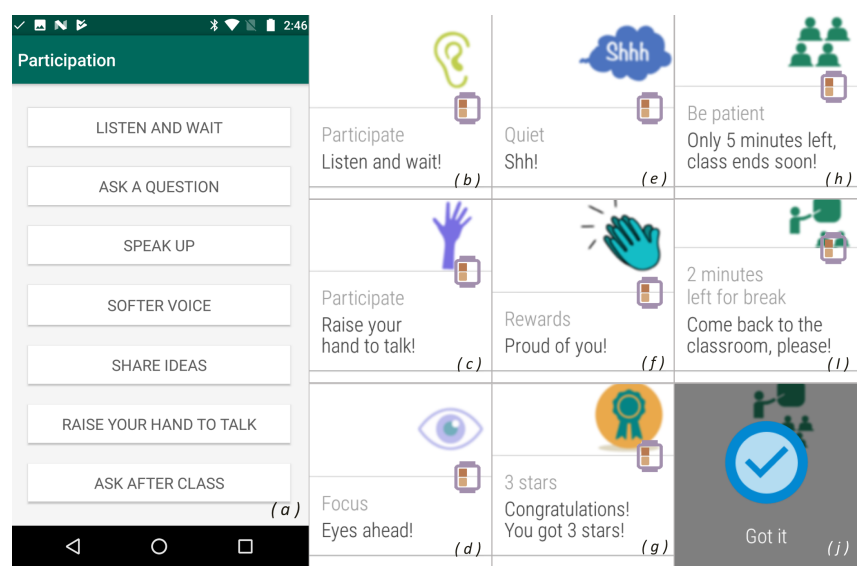

Figure 2. User interfaces for the mobile application and for the smartwatch application: (a) menu with seven options for participation; (b, c) two notifications on watch about participation; (d) a notification on watch about Focus; (e) a notification on watch about Quiet; (f, g) two watch notifications about Rewards; (h) an Alarm notification for the watch; $(i, j)$ a countdown notification and confirmation screen for the watch application.

cations, for example the extent and strength of the vibration of the watch.

To guide the graphic design and decide on a main character for WELI (avatar-like), we conducted an online survey asking the assistants about their preferences. We provided eight examples of design illustrations to help decide whether to employ abstract or cartoonish characters. The majority of the participants (assistants) preferred a more abstract style. Further design considerations included having a neutral character, in terms of age, gender, race and ethnicity.

\section{Phase 3: User Studies}

Terminology Survey

To get feedback on the terminology used in WELI, we held a participatory design session with 32 assistants of Mason LIFE. For the target user populations-students with IDDs and their assistants-terminology choice is key to facilitating ease of use and learning. We demonstrated WELI 1 using a phone and a watch and presented a video showing all the features and the interaction steps of the application. To assess the terminology used and gather feedback about it, we conducted a survey comprising 35 questions, among which 29 covered the terminology used for the menu items, main features and sub features, and 4 focused on interaction, graphic design, and devices. The two last questions were open for comments and suggestions. Each question was judged as: appropriate, neutral, or inappropriate, and allowed for comments for justification purposes or suggestions of alternative terms.

\section{Updates in Terminology and Features}

Concerning the first 29 questions, $66 \%$ of the terms used were classified as 'appropriate', $24 \%$ as 'neutral' and $10 \%$ as 'inappropriate'. Overall, the initial terms were considered as acceptable. Treating each question separately, and comparing the number of the choices considered 'appropriate' with the ones considered 'neutral' or 'inappropriate', $72 \%$ of the terms used were rated 'appropriate', and $28 \%$ of the terms were considered 'neutral' or 'inappropriate', indicating the specific words that were considered unclear or inappropriate. A recurrent comment and common suggestion from the assistants was for wording to be "more specific", "better defined" and "simplified but not vague”.

Based on the results obtained, we changed the terminology employed for WELI 2 (WELI app version 2). To be more specific, we maintained the words considered as highly 'appropriate'. "Stop and listen" for instance was considered 'appropriate' by $89 \%$ of participants. Conversely, "concentrate" received only $38 \%$ 'appropriate' responses, thus we replaced it with three more concrete expressions as suggested: "eyes ahead", "listen carefully" and "put the phone away". Likewise, we replaced the general expression "Start to participate" with a more specific expression: "Raise your hand to talk" and "Share ideas", in the Participation feature. We also changed the names of the features. "Discussion" was replaced with "Participation" as recommended in the survey responses. We removed negative words, like "inappropriate".

As reinforced in the survey responses, the students with IDDs are already trained to employ the "Zones of Regulation" [15] for self-regulation of their affective states. Thus, we synchronized the application terminology to be consistent with the training the students already receive. First, we replaced the feature named "mood" to "self-assessment". Then, to be more specific within the 'self-assessment' feature, we replaced the choice of specific moods ('stressed', 'bored', 'happy', 'tired') used in WELI 1, with four color zones ('red', 'yellow', 'green', and 'blue') pertaining to the Zones of Regulation. Each color zone expresses an affective state, rather than a specific mood, and the students are already accustomed to use the four color zones for self-regulation. The graphic icon designs were updated accordingly. In the survey, the assistants considered that the icon initially employed was similar to a "baby" boy and suggested to replace it with an "emoji"-like icon. Thus, for WELI 2, we updated the icons to represent the facial expressions and set of colors corresponding to the regulation zones (red, yellow, green, blue). The updated design, similar to "emojis", was considered to be more inclusive and gender-neutral.

As the assistants emphasized in the user studies, "positive reinforcement" is essential to improve the students' engagement in special education. Given that "all students benefit" from and "love all the positive feedback", we added a "rewards" feature in WELI 2. This feature enables the assistants to send messages such as "proud of you", "awesome", and "thumbs up". For each message received, the student accumulates points based on the number of rewards received in class. An 'after class survey' was also added as a feature, so that the assistants could gather the students' feedback about each class.

\section{Usability Study}

To assess WELI 2 after updating the terminology employed and extending its features, we conducted two usability study sessions. The study participants included students and the assistants organized in pairs, with six assistants (four students and two professors in special education) and seven students with IDD (four in the first session and three in the second one). 


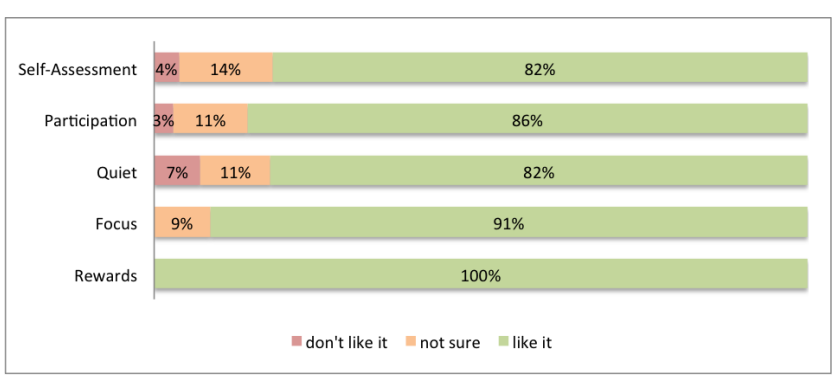

Figure 3. Histogram of the students' responses in the usability study for self-assessment, participation, quiet, focus and rewards features

To moderate the sessions, the study was coordinated by one developer, one professor in HCI and 1 graphic designer who observed, documented and took notes about the sessions. The seven students composed a gender-balanced sample, being three male and four female; also, concerning their graduation year, four were sophomores, two alumni and one senior. Two students wore the watch on their left wrist and five on the right one. The average age of the participants was 24 years old.

The usability study aimed at assessing five in-class features, namely: 'focus', 'quiet', 'participation', 'self assessment' and 'rewards'. Before each session, we briefly introduced the study protocol and the application, and presented a video to the assistants and to the students to demonstrate WELI. Then, one assistant and one student worked together to mimic an interactive scenario in class, simulating different WELI features in which the assistant sends a notification by phone to the watch of the student. To ensure the completion of a consistent protocol, all participants' pairs (assistant and student) tested all the features available in the app. After each feature was tested, the student assessed whether he/she "liked it", "was not sure" or "didn't like it", selecting one response. Then, the assistants filed a paper form accordingly for record keeping. The student was also inquired about their considerations and any additional comments to improve the features. All the sessions were video-recorded.

To analyze the user interaction comparing two approaches for input entry on a smartwatch, the watch app logged the students' input for self-assessment. We employed horizontal swipe to select 'zones' (slide the watch screen right or left to select one 'color' button for the choice of affective state) and vertical scroll to select specific 'levels' of the regulation zone previously selected. The students had not received any training to learn how to interact with a smartwatch. All the students who participated in the study did not have their a smartwatch of their own.

\section{Findings}

In total, we gathered 301 (7X43) responses from seven students. Only seven responses were "Don't like it"(2.3\%), while 28 referred to "Not Sure" $(9.3 \%)$, and 266 were "Like $i t$ " $(88.4 \%)$, indicating a positive acceptance in general and a potential acquiescence bias (Figure 3). The results per feature are described as follows:

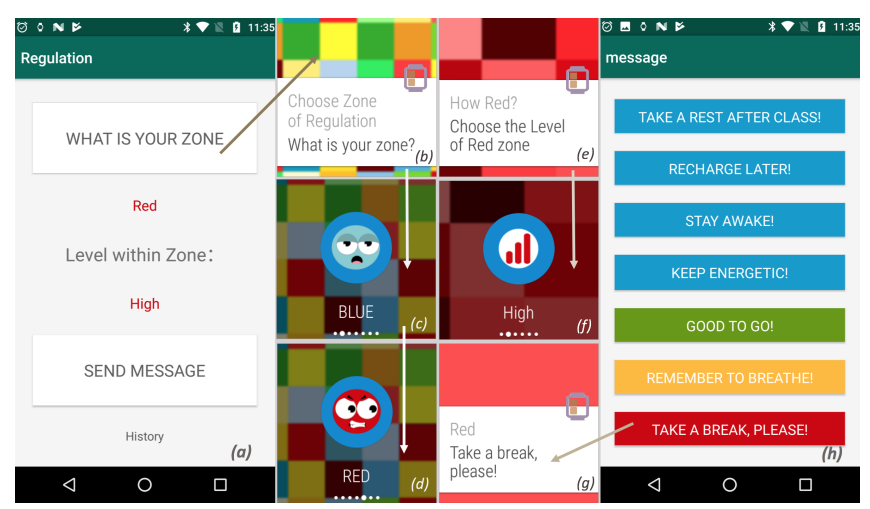

Figure 4. User interfaces for self-assessment. In this example, the Red (d) zone is chosen as a response for question regarding the type of zone (b), and the level high is chosen (f) concerning the level question within the red zone (e). In (a) the results of the zone and level are shown on the phone, and a specific message (g) is sent through the mobile application (h) for intervention according to the student response. The arrows between user interfaces indicate the interaction path.

- Rewards: all the features within rewards got responses of "Like it", from the seven students.

- Focus: $91.4 \%$ got "Like it" as responses, $8.6 \%$ got "Not sure" as response.

- Quiet: $82.2 \%$ got "Like it" and 10.7\% got "Not sure", 7.1\% "Don't like it".

- Participation: $85.7 \%$ got "Like it" and $11.4 \%$ got "Not sure", $2.9 \%$ "Don't like it".

- Self Assessment: $81.9 \%$ got "Like" and 14.3\% got "Not sure", $3.8 \%$ "Don't like it".

Rewards was considered as the most favorable feature for all students, followed by Focus, the second most relevant feature and without any dislike response. The other three features received similar responses, however among them, the Quiet feature received more dislike responses (7.1\% dislike). Concerning the Rewards feature, the students liked it because it made them "feel happy", "proud" and "independent" as well.

Comparing the interaction styles in the analysis of the log files, the 'zone of regulation' (horizontal swipe) had only one mistake documented among 28 interactions ( 7 students $\times 4$ zones) which was caused by a confusion between the green and the blue zone. Despite the two colors being similar, the green icon presented a happy expression and also had the word 'green' displayed below it. The blue icon (corresponding to 'bored') also had a 'blue' label. Still, we noticed that the design was not distinctive enough for the students to select the right color at a glance. As a design implication, we made the icons' colors even more distinguishable and also exaggerated the facial expression as shown in Figure $4 \mathrm{c}$ and $\mathrm{d}$, to reinforce the graphic icons associated to the zones of regulation.

For the 'level' of regulation (vertical scroll for selection), there were six mistakes made among 28 operations. Besides one duplicated touch, all the other mistakes (5 out of 6 ) were made 
by 3 students who selected the microphone icon displayed on the top of screen (Figure $5(\mathrm{~g})$ ). The microphone is a default option in Android Wear to use voice commands as input entry. In those occasions, we noticed that the students started to speak to their watches, and the log file recorded the scripts of their speeches. The results demonstrate that the students actually liked this feature, for example one student mentioned: "Wow, I am speaking to the watch!", as recorded in the log file. Also, one assistant mentioned in her notes: "P2 was able to scroll and responded enthusiastically to each message. She was also able to find the voice control instead of the rating scale.". However, using voice commands as input for selfassessment is not ideal, given that when the students speak to the watch in class, they disturb their peers and it is also distracting to themselves. The comments provided by the five assistants indicate that they do not want the students to speak to their watches during class.

In addition to this, the assistants also recommended the selection of the 'level' of the regulation zone to employ the same interaction approach as the one available for selecting a 'zone of regulation' (i.e. a horizontal swipe). The assessment of 'level' is displayed sequentially right after the selection of a regulation 'zone'. Completing both interactions with a horizontal swipe would ensure consistency in the self-assessment feature, making it easier to learn and to use. Thus, the updated version of the application, WELI 3, employs horizontal swipes for input entry for both 'zones' and 'levels'. To facilitate the user interaction, WELI 3 also triggers the 'level' question automatically after receiving the response of the type of 'zone' selection.

In summary, by incorporating the analysis of the interaction logs with the application, we optimized the graphic design to better convey information and deliver messages to the students. We ensured that the studentâĂŹs interaction with the application became more consistent, and facilitated the operations performed by the assistant with a more automated approach. Those were the main updates implemented in WELI 3.

Finally, we asked the students what they think about the smartwatch, right before and after the study. Before the study and using the watch, the students watched a video about WELI, and their comments were positive overall, including: "Pretty good-looking", "It helps", and "It is very cool". Only two students had no comments. For the assistants, the comments were also positive overall, including: "It will be very efficient for students to tell how they feel by looking at the watch".

After the study, all the students chose "Like" as the response, and all the students had positive comments and feedback, for instance: "I love it, I love the watch", "Because I'm happy; because I like this smart watch", "Good, great, can't wait to try it out", "Feels good, feels light", "It would help because I know when to settle down, I can participate and listen to the teacher", "Good" and "I think it will be very helpful for students in their Exploration class because it will control how they are feeling". The students' reactions turned out to be more positive after the study, as by having experienced WELI, they became more excited and enthusiastic about the technology.
WELI

WELI is implemented as an an application for Android phones (Moto Plus 5G and LG Nexus 5X) using Android Wear (Sony Smartwatch 3 SWR50) with Android 7.0 Nougat and Android Wear 1.5. The devices are connected via Bluetooth, which is sufficient for assistants to send notifications to the student watch in a classroom. Notifications are sent automatically (preprogrammed) or manually triggered by the interaction of the assistant via a mobile application. With ten features available, WELI helps the assistants to provide timely support to the students with an unobtrusive and consistent approach. WELI is intended to enable assistants to provide timely support to students in an unobtrusive and consistent fashion to minimize in-class distraction and foster student engagement.

\section{Mediation}

WELI offers mediation features of 'Focus', 'Quiet', and 'Participation' which provide intervention options to keep students more focused (Figure 2 (d)), quiet (Figure 2 (e)) (when the student is talking too much or too loud), and engaged in classroom activities. Each of these features offers the assistants several choices of messages to be sent to the student's watch, according to the specific context. For example, 'Participation' includes options to 'listen and wait', 'ask a question', 'softer voice', and 'raise your hand to talk' (Figure 2 (a), (b), and (c)). For each option, a notification message entitled "Participate" is displayed on the student's watch. The student is notified through a short vibration of the watch, so that he/she can glance at the UI contents and read the text message. To minimize distraction, these three features are passive, i.e. they do not require any explicit interaction from the student.

\section{Rewards}

WELI offers assistants a 'rewards' feature to provide positive reinforcement to students. The message options and interactions are similar to the three features previously presented (Figure 2 (f)). However, it also includes a function to accumulate the rewards earned by the student in class (Figure $2(\mathrm{~g})$ ), a function to send messages to the student, and a function for the assistants to track the student's progress.

\section{Self-Assessment}

WELI offers assisants a self-assesment feature to provide the 'Zones of Regulation' [15] on the student's watch. Zones of Regulation is a theoretical framework largely adopted by the students with IDDs in Mason LIFE for them to periodically self-assess and regulate their affective states. The selfassessment feature requires the student to slide the UI horizontally and select an appropriate response, to then receive the respective intervention (Figure 4). This feature includes two steps. The $1^{\text {st }}$ step consists of the assistant selecting 'what is your zone' (Figure 4 (a)) as a question, triggering an immediate notification in the student's watch application asking about his/her current affective zone (Figure 4 (b)). To respond to the question, the student slides the notification to the right and selects one of the icons corresponding to his/her affective state. The student can choose among four possible regulation zones (Red, Yellow, Green and Blue) (Figure 4 (c), (d)). The student response is sent to the assistant's phone (Figure 4 (a)). 


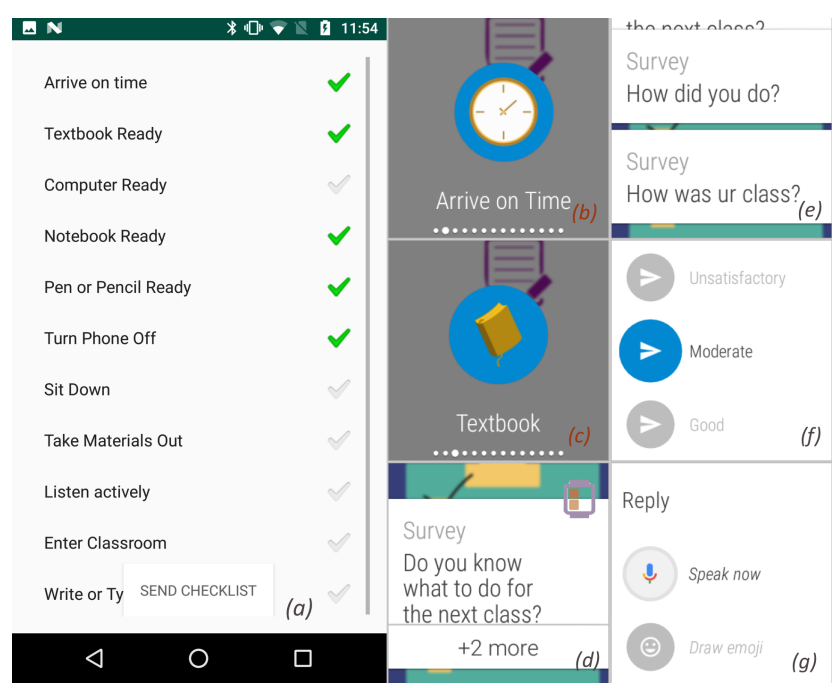

Figure 5. User interfaces for the Readiness Checklist and After Class Survey. For Checklist, the student selected for instance the items (b) and (c) that they have already prepared. The corresponding items are also checked on the assistant's phone (a). For Survey, a stack of notifications, which can display the 3 questions at once is shown in (d), (e) and the survey answers to choose vertically is shown in (f), (g), and the (f) is the choice to answer the question 'How did you do?'

The $2^{\text {nd }}$ step asks the student about the level of the chosen zone where he/she is located (Figure 4 (c)). The notification about the zone level (Figure 4 (e)) is automatically triggered after the response has been sent to the assistant, and allows the student to choose among 'high', 'medium' and 'low' levels (Figure 4 (f)). After the assessment is completed by the student, the assistant sends another message-the intervention-which helps the student according to the zone and level he/she selected (Figure $4(\mathrm{~h}),(\mathrm{g})$ ).

\section{Readiness Checklist}

The readiness checklist is sent to the student a few minutes before the class starts to aid them to be prepared for class. The students can slide the notification to the right to navigate through a procedural checklist and select the preparation status according to what they have already done. The actions checked are automatically updated on the assistant's mobile application, as shown in Figure 5 (a), (b), and (c).

\section{After-Class Survey}

The after-class survey feature aims at gathering feedback from students about a given class. The assistant sends a survey after the end of a class, and the student receives three questions at once (on a single screen), as stacked notifications (Figure 5 (d), (e)). To answer the questions, the student selects the notifications of the stack one-by-one. For each question, the student can choose a single answer by sliding right and selecting a reply, then scrolling vertically to choose one answer among three options available (Figure 5 (f)). Besides the three fixed choices provided, the vertical interaction also allows for voice input (Figure $5(\mathrm{~g})$ ), enabling the students to speak after class to provide comments and more detail about their classroom experiences.

\section{Scheduling}

The countdown and alarm features enable the assistant to set reminders for students. The assistant can set a few minutes to countdown for a class break or for a drink/snack break. When the break time is over, a notification is sent to the student informing that he/she should come back to the classroom (Figure 2 (i)). The student can slide the notification to the right and select the blue icon (Figure $2(\mathrm{j})$ ) to inform the assistant that they received the message and they will come back to the classroom. For acknowledgment purposes, the student selection triggers a short message in the assistants' phone informing them that the student received the message ('got it' is displayed). With the alarm feature, the assistant can set notifications (Figure $2(\mathrm{~h})$ ) to be repeated weekly at a certain time. These notifications are sent automatically to the student.

\section{Settings}

The 'settings' feature enables the assistant to use a switch button to customize the vibration of the notifications as 'strong' or 'mild'. The assistant can also click on the share log button in settings to automatically launch an email application (Gmail) and send an email message to the assistant containing the $\log$ file of the interaction attached for later analysis and visualization. The file includes the log of the user interaction related to self-assessment and after class survey features. The $\log$ files help the assistant to monitor the student outcomes in a timely, individualized approach.

\section{DESIGN CONSIDERATIONS}

A limited number of user studies have been conducted with students with IDDs, thus the benefits of emerging technologies to support them in post-secondary education are not yet well-understood. Further, as this user population has not been largely studied by the HCI community, traditional usercentered approaches are not fully appropriate to design interactive solutions for them. To conduct the user studies in our research project, we adapted existing techniques to consider the special needs of students with IDDs. The four main design considerations taken concerned assistants, data collection, study protocol, and ethics. Those are described as follows.

As usual, the user studies conducted, including focus groups, interviews, and usability tests, were lead by HCI experts. However, all studies were also conducted with the presence of at least one assistant who is expert in special education. The assistants are already familiar with the students' population and helped to re-phrase and elaborate questions when the students were not following the study protocol or misunderstood the instructions given. The assistants were also essential in the studies to monitor and mediate the students' behaviors whenever necessary, helping them to become more engaged with the activities proposed.

To collect data and the students' feedback, we adopted simplified surveys. The questions were brief (short statements) and the language straight-forward. We used 3-point scales with semantic differential for the possible answers, for instance: I like, I don't like it, I don't know. We also relied on conventional graphic representations to reinforce the meaning of 
possible responses, using a smiley emoticon, thumbs up, and thumbs down as icons to convey different satisfaction levels.

The study protocol was defined in collaboration with special education experts in order to ensure that the terminology of the questions would facilitate the students' comprehension and the protocol employed would foster their attention throughout the study session. The sessions were short to prevent fatigue and facilitate sustained engagement (60 minutes at most).

To preserve the students' privacy only the sessions conducted in controlled settings (not during regular classes) were documented with audio and video. The contents were transcribed verbatim for data analysis. The log files did not include any personally-identifiable information.

\section{DISCUSSION}

Wrist-worn technologies, such as Apple Watch and Fitbit, have become more popular in recent years. However, most commercial devices are employed as fitness trackers and little work has been done to investigate the application of smartwatches as assistive technologies. To explore the potential of wearable technologies for students with IDDs in an inclusive special education program, we conducted a 2 -year research project following an iterative, user-centered design approach. The design requirements were elicited in eight user studies involving 58 participants in total-students with IDDs, assistants, experts in Special Education and in HCI. To design and develop WELI, we employed Android Phones (Moto Plus 5G and LG Nexus 5X) and Android Wear Smartwatches (Sony Smartwatch 3 SWR50). WELI aims at not only helping students with IDDs in class, but also facilitating the work of their assistants. Thus far, the assistants and the students with IDDs involved in the project provided positive feedback about WELI.

Despite WELI receiving a positive reaction from both students and assistants in preliminary evaluations, the WELI application has some considerations and limitations. Long-term field studies are needed to validate and triangulate the initial responses given by students, as their responses may be subject to bias related to acquiescence or novelty. The assistants, despite being receptive to the solution, expressed several concerns about students with IDDs using a wearable application in class. Assistants stated that they “...just don't want the students to get frustrated if the watch doesn't work....”. They feared that "the students may lose the watch"; and they also expressed concerns that "the watch may cause a distraction to some students, who are addicted to their tech and devices.". In addition to those, they also raised concerns about balancing a universal solution and a personalized design, mainly because, as one assistant mentioned " all of our students are unique individuals". Lastly, there were also concerns about the privacy of the students with IDDs, to not disclose their sensitive personal information when the data was continuously monitored.

Concerning the limitations of WELI, the application developed is platform-specific, built with Android technologies. As future work, we plan to release an iOS version for Apple watches. WELI focuses on helping students with IDDs mainly in-class, supporting interactive activities during a class and a few minutes before and after it. Although the class is the most important activity in academic life, students with IDDs need help beyond the classroom environment, in and out of the college campus. As future work, we plan to enable students to review class contents of a class and do their homework at home with support from WELI. Additional activities that will be considered in future releases include borrowing books from the library and getting the university shuttle. The main constraint associated to the hardware is related to the short battery life of the smartwatch $(48 \mathrm{~h})$, which remains an important limitation.

\section{CONCLUSION}

To assist young adults with intellectual and developmental disabilities (IDDs) and help them to become more independent and engaged in a post-secondary inclusive program, we designed and developed WELI. WELI is available for Android smartphones and Android smartwatches, serving as a complementary tool for assistants who send wearable notifications to the students using a mobile application. The features provided include reminders for focus, silence, participation, rewards, countdown, checklist, survey and self-assessment. The application requirements were elicited and refined through eight user studies conducted with students with IDDs, their assistants and experts in special education. By providing help to the students and facilitating the work of the assistants, WELI enables students to depend less on the assistants in class, improving their self-confidence. The results of our usability study reveal that designing for students with IDDs require special considerations to accommodate their needs, but also that students are excited about using novel wearable technologies in class to assist them. In addition to that WELI requires no training for the students to learn how to interact with the watch. All the features provided by WELI received positive feedback, particularly the rewards feature.

As future work, we will conduct long-term field studies involving students and assistants to better assess how WELI impacts their daily routines in inclusive classes, to gather additional feedback about the application. We will also add features to help the students with IDDs to give a presentation in class and to help the assistants to take notes. Further customization options will be added for personalization. Moreover, we will explore the use of the watch sensors to provide smart and automated support, for instance to suggest breaks for the students when they are feeling sleepy in class. Finally, we plan to extend WELI to cover activities outside of the class.

\section{ACKNOWLEDGMENTS}

We thank all the participants of the user studies and the Mason LIFE staff for facilitating this research, especially Dr. Anya Evmenova, Dr. Heidi Graff and Kudirat O Giwa. We thank George Mason University for the financial support, in special the Oscar Program, Alaa Zabara for designing the graphics, Serge Magnavox and Pattiya Mahapasuthanon for aiding with the video. This material is based upon work supported by the National Science Foundation under Grant No.1314342. Any opinions, findings, conclusions or recommendations expressed in this material are those of the author(s) and don't necessarily reflect the views of the National Science Foundation. 


\section{REFERENCES}

1. LouAnne E. Boyd, Xinlong Jiang, and Gillian R. Hayes. 2017. ProCom: Designing and Evaluating a Mobile and Wearable System to Support Proximity Awareness for People with Autism. In Proceedings of the 2017 CHI Conference on Human Factors in Computing Systems (CHI '17). ACM, New York, NY, USA, 2865-2877. DOI : http://dx.doi.org/10.1145/3025453.3026014

2. Coleen A Boyle, Sheree Boulet, Laura A Schieve, Robin A Cohen, Stephen J Blumberg, Marshalyn Yeargin-Allsopp, Susanna Visser, and Michael D Kogan. 2011. Trends in the prevalence of developmental disabilities in US children, 1997-2008. Pediatrics 127, 6 (2011), 1034-1042.

3. Luke Buschmann, Lourdes Morales, and Sri Kurniawan. 2014. Online Learning System for Teaching Basic Skills to People with Developmental Disabilities. In Proceedings of the 16th International ACM SIGACCESS Conference on Computers \& Accessibility (ASSETS '14). ACM, New York, NY, USA, 271-272. DOI : http://dx.doi.org/10.1145/2661334.2661391

4. Karina Caro, Ana I. Martínez-García, Mónica Tentori, and Iván Zavala-Ibarra. 2014. Designing Exergames Combining the Use of Fine and Gross Motor Exercises to Support Self-care Activities. In Proceedings of the 16th International ACM SIGACCESS Conference on Computers \& Accessibility (ASSETS '14). ACM, New York, NY, USA, 247-248. DOI : http://dx.doi.org/10.1145/2661334.2661403

5. Yao-Jen Chang, Ya-Shu Kang, Yao-Sheng Chang, Hung-Huan Liu, Cheng-Chieh Wang, and Chia Chun Kao. 2015. Designing Kinect2Scratch Games to Help Therapists Train Young Adults with Cerebral Palsy in Special Education School Settings. In Proceedings of the 17th International ACM SIGACCESS Conference on Computers \&\#38; Accessibility (ASSETS '15). ACM, New York, NY, USA, 317-318. DOI : http://dx.doi.org/10.1145/2700648.2811356

6. Victor Dibia. 2016. FOQUS: A Smartwatch Application for Individuals with ADHD and Mental Health Challenges. In Proc. of ASSETS'16. USA, 311-312. DOI : http://dx.doi.org/10.1145/2982142.2982207

7. Victor Dibia, Shari Trewin, Maryam Ashoori, and Thomas Erickson. 2015. Exploring the Potential of Wearables to Support Employment for People with Mild Cognitive Impairment. In Proc. of ASSETS'15. Portugal, 401-402. DOI:

http://dx.doi.org/10.1145/2700648.2811390

8. Alexander Fiannaca, Ilias Apostolopoulous, and Eelke Folmer. 2014. Headlock: A Wearable Navigation Aid That Helps Blind Cane Users Traverse Large Open Spaces. In Proceedings of the 16th International ACM SIGACCESS Conference on Computers \& Accessibility (ASSETS '14). ACM, New York, NY, USA, 19-26. DOI : http://dx.doi.org/10.1145/2661334.2661453

9. Euan Freeman, Graham Wilson, Stephen Brewster, Gabriel Baud-Bovy, Charlotte Magnusson, and Hector
Caltenco. 2017. Audible Beacons and Wearables in Schools: Helping Young Visually Impaired Children Play and Move Independently. In Proceedings of the 2017 CHI Conference on Human Factors in Computing Systems (CHI '17). ACM, New York, NY, USA, 4146-4157. DOI : http://dx.doi.org/10.1145/3025453.3025518

10. Markus Funk, Sven Mayer, and Albrecht Schmidt. 2015. Using In-Situ Projection to Support Cognitively Impaired Workers at the Workplace. In Proceedings of the 17th International ACM SIGACCESS Conference on Computers \&\#38; Accessibility (ASSETS '15). ACM, New York, NY, USA, 185-192. DOI : http://dx.doi.org/10.1145/2700648.2809853

11. Dorothy F. Garrison-Wade. 2012. Listening to their voices: Factors that inhibit or enhance postsecondary outcomes for students with disabilities. International Journal of Special Education 27, 2 (2012), 113-125. DOI : http://dx.doi .org/10.1177/089443939201000402

12. Dedra Hafner, Courtney Moffatt, and Nutullah Kisa. 2011. Cutting-Edge. Career Development for Exceptional Individuals 34, 1 (2011), 18-30. DOI : http://dx.doi.org/10.1177/0885728811401018

13. Stefan Johansson, Jan Gulliksen, and Ann Lantz. 2015. User Participation When Users Have Mental and Cognitive Disabilities. In Proceedings of the 17th International ACM SIGACCESS Conference on Computers \&\#38; Accessibility (ASSETS '15). ACM, New York, NY, USA, 69-76. DOI : http://dx.doi.org/10.1145/2700648.2809849

14. Thomas Kosch, Romina Kettner, Markus Funk, and Albrecht Schmidt. 2016. Comparing Tactile, Auditory, and Visual Assembly Error-Feedback for Workers with Cognitive Impairments. In Proceedings of the 18th International ACM SIGACCESS Conference on Computers and Accessibility (ASSETS '16). ACM, New York, NY, USA, 53-60. DOI : http://dx. doi .org/10.1145/2982142.2982157

15. Leah Kuyper. 2014. the Zones of reglation. website. (2014). Retrieved Sept. 2nd, 2017 from http://www . zonesofregulation. com/index.html.

16. Stephen Lipscomb, Joshua Haimson, Albert Y. Liu, John Burghardt, David R. Johnson, and Martha L. Thurlow. 2017. Preparing for life after high school: The characteristics and experiences of youth in special education. Findings from the National Longitudinal Transition Study 2012. Volume 1: Comparisons with other youth: Full report. (03 2017).

17. Sinziana Mazilu, Ulf Blanke, Michael Hardegger, Gerhard Tröster, Eran Gazit, and Jeffrey M. Hausdorff. 2014. GaitAssist: A Daily-life Support and Training System for Parkinson's Disease Patients with Freezing of Gait. In Proceedings of the 32Nd Annual ACM Conference on Human Factors in Computing Systems (CHI '14). ACM, New York, NY, USA, 2531-2540. DOI : http://dx.doi.org/10.1145/2556288.2557278 
18. Lourdes M. Morales-Villaverde, Karina Caro, Taylor Gotfrid, and Sri Kurniawan. 2016. Online Learning System to Help People with Developmental Disabilities Reinforce Basic Skills. In Proceedings of the 18th International ACM SIGACCESS Conference on Computers and Accessibility (ASSETS '16). ACM, New York, NY, USA, 43-51. D0I :

http://dx.doi.org/10.1145/2982142.2982174

19. Nathalie Nader-Grosbois. 2014. Self-perception, self-regulation and metacognition in adolescents with intellectual disability. 35 (04 2014), 1334-1348.

20. Wagner M. Cameto R. Knokey A. M. Shaver D. Newman, L. 2010. Comparisons across Time of the Outcomes of Youth with Disabilities up to 4 Years after High School. A Report of Findings from the National Longitudinal Transition Study (NLTS) and the National Longitudinal Transition Study-2 (NLTS2). NCSER 2010-3008. (September 2010).

21. American Association on Intellectual and Developmental Disabilities. 2010. Intellectual Disability: Definition, Classification, and Systems of Supports. American Association on Intellectual and Developmental Disabilities.

https://books. google. com/books?id=0g8-QQAACAAJ

22. Kathryn E. Ringland, Christine T. Wolf, LouAnne E. Boyd, Mark S. Baldwin, and Gillian R. Hayes. 2016. Would You Be Mine: Appropriating Minecraft As an Assistive Technology for Youth with Autism. In Proceedings of the 18th International ACM SIGACCESS Conference on Computers and Accessibility (ASSETS '16). ACM, New York, NY, USA, 33-41. DOI: http://dx.doi.org/10.1145/2982142.2982172

23. Robert L Schalock. 2010. Intellectual disability. The Encyclopedia of Clinical Psychology (2010).

24. Sumita Sharma, Saurabh Srivastava, Krishnaveni Achary, Blessin Varkey, Tomi Heimonen, Jaakko Hakulinen, Markku Turunen, and Nitendra Rajput. 2016. Gesture-based Interaction for Individuals with Developmental Disabilities in India. In Proceedings of the 18th International ACM SIGACCESS Conference on Computers and Accessibility (ASSETS '16). ACM, New York, NY, USA, 61-70. DOI : http://dx.doi.org/10.1145/2982142.2982166

25. Will Simm, Maria Angela Ferrario, Adrian Gradinar, Marcia Tavares Smith, Stephen Forshaw, Ian Smith, and Jon Whittle. 2016. Anxiety and Autism: Towards Personalized Digital Health. In Proc. of CHI'16. USA, 1270-1281. DOI: http://dx.doi.org/10.1145/2858036.2858259
26. Kiley Sobel, Kyle Rector, Susan Evans, and Julie A. Kientz. 2016. Incloodle: Evaluating an Interactive Application for Young Children with Mixed Abilities. In Proceedings of the 2016 CHI Conference on Human Factors in Computing Systems (CHI'16). ACM, New York, NY, USA, 165-176. DOI : http://dx.doi.org/10.1145/2858036.2858114

27. Tobias Sonne, Jörg Müller, Paul Marshall, Carsten Obel, and Kaj Grønbæk. 2016. Changing Family Practices with Assistive Technology: MOBERO Improves Morning and Bedtime Routines for Children with ADHD. In Proceedings of the 2016 CHI Conference on Human Factors in Computing Systems (CHI'16). ACM, New York, NY, USA, 152-164. DOI : http://dx.doi.org/10.1145/2858036.2858157

28. Vicky Spencer, Anna Evmenova, Richard T. Boon, and Laura Harris. 2014. A review of research-based interventions for students with Autism Spectrum Disorders (ASD) in content area instruction: Implications and considerations for classroom practice. 49 (09 2014), $331-354$

29. Kenji Suzuki, Taku Hachisu, and Kazuki Iida. 2016. EnhancedTouch: A Smart Bracelet for Enhancing Human-Human Physical Touch. In Proc. of CHI'16. USA, 1282-1293. DOI :

http://dx.doi.org/10.1145/2858036.2858439

30. Camellia Zakaria and Richard C. Davis. 2016. Demo: Wearable Application to Manage Problem Behavior in Children with Neurodevelopmental Disorders. In Proc. of MobiSys'16. Singapore, 127-127. DOI : http://dx.doi.org/10.1145/2938559.2938575

31. Xiaoyi Zhang, Harish Kulkarni, and Meredith Ringel Morris. 2017. Smartphone-Based Gaze Gesture Communication for People with Motor Disabilities. In Proceedings of the 2017 CHI Conference on Human Factors in Computing Systems (CHI '17). ACM, New York, NY, USA, 2878-2889. DOI : http://dx.doi.org/10.1145/3025453.3025790

32. Hui Zheng and Vivian Genaro Motti. 2017a. Wearable Life: A Wrist-Worn Application to Assist Students in Special Education. In International Conference on Universal Access in Human-Computer Interaction. Springer, 259-276.

33. Hui Zheng and Vivian Genaro Motti. 2017b. WeLi: A Smartwatch Application to Assist Students with Intellectual and Developmental Disabilities. In Proceedings of the 19th International ACM SIGACCESS Conference on Computers and Accessibility. ACM, 355-356. 